\title{
Toward maximizing immunotherapy in metastatic castration-resistant prostate cancer - rationale for combinatorial approaches using chemotherapy
}

\author{
Susan R. Slovin* \\ Genitourinary Oncology Service, Sidney Kimmel Center for Prostate and Urologic Cancers, Memorial Sloan-Kettering Cancer Center, New York, NY, USA
}

\section{Edited by:}

Derek Raghavan, Carolinas

HealthCare System, USA

Reviewed by:

Derek Raghavan, Carolinas HealthCare System, USA

David lan Quinn, University of Southern California Kenneth J. Norris Jr. Comprehensive Cancer Center, USA

Chao-Nan (Miles) Qian, Van Andel Research Institute, USA

*Correspondence:

Susan R. Slovin, Genitourinary Oncology Service, Sidney Kimmel Center for Prostate and Urologic

Cancers, Memorial Sloan-Kettering Cancer Center, 1275 York Avenue,

New York, NY 10065, USA.

e-mail: slovins@mskcc.org
Prostate cancer is particularly suited for active immunotherapy because of the expression of a distinctive number of antigens which are overexpressed on prostate cancer cells and cell lines. There is evidence in this disease that tumors promote immune tolerance starting early in the disease course. As such, chemotherapy, by suppressing tumors and activating immune system homeostatic mechanisms, may help overcome this tumor-induced immune tolerance. Sipuleucel-T which has recently been approved in the US, is an autologous cellular product immunotherapy that induces immune activity likely through activation of dendritic cells. This was associated with a survival benefit in the absence of significant toxicity. However, a post hoc analysis of phase III trial participants found a substantial survival benefit to receiving docetaxel some months after sipuleucel-T. However, another phase III immunotherapy trial combining a prostate cancer therapeutic vaccine GVAX plus docetaxel versus standard docetaxel therapy in advanced prostate cancer, observed a lower overall survival with the vaccine regimen. These trials highlight major unresolved questions concerning the optimum choice, dosing, and timing of chemotherapy relative to active immunotherapy and the overall merits of considering this approach. The ideal treatment approach remains unclear; advances in biomarker validation and trial design may likely improve our ability to assess biologic benefit irrespective of the development of true antitumor immunity.

Keywords: prostate cancer, chemotherapy, vaccines, prostate specific antigen, prostate specific membrane antigen

\section{INTRODUCTION}

Therapeutic options for the treatment of castration-resistant prostate cancer (CRPC) have dramatically improved with the approval of three new treatments each of which is unique in its ability to target a particular mechanism within the spectrum of prostate cancer growth. While docetaxel remains the first-line standard of care for metastatic CRPC due to its demonstrated survival benefit, improved pain control and quality of life, cabazitaxel, a semi-synthetic derivative of the 10-deacetylbaccatin III that promotes tubulin assembly in vitro and stabilizes microtubules against cold-induced depolymerization, salvaged docetaxel failures with benefit of antitumor effects and a small survival advantage. Abiraterone (Zytiga ${ }^{\mathrm{TM}}$ ), an inhibitor of CYP-17, recently received FDA approval as another post-docetaxel second-line agent, and as seen with cabazitaxel (Jevtana ${ }^{\mathrm{TM}}$ ), these agents offered about a 4-month survival benefit, respectively. For the first time, immunotherapy, a long-sought after treatment approach that has been fraught with suboptimal results has finally come of age in the form of active cellular product, Sipuleucel-T, also associated with a survival benefit. While there are data to suggest that the first two treatments are well-placed in the post-docetaxel setting, their entry into earlier clinical states of prostate cancer growth is being investigated. Sipuleucel-T remains for castration-resistant disease at any stage.
The rationale for immunotherapy in prostate cancer has been based on several observations. First, there are a several altered selfantigens, glycoproteins, and glycolipids, which are overexpressed or underglycosylated on prostate cancer cells and cell lines (Slovin, 2008). These include the mucins MUC-1, 2, Globo H, GM-2, prostate stem cell antigen (PSCA), prostate specific antigen (PSA), and prostate specific membrane antigen (PSMA). PSMA has been a target for a variety of approaches including protein and peptide vaccines, naked DNA vaccines, chemotherapy-anti-PSMA monoclonal antibody conjugates, as well as radiolabeled anti-PSMA monoclonal antibodies. Second, a biomarker PSA, can be used to monitor disease activity and/or progression. Third, prostate cancer can have a long natural history, thus all clinical states can be potentially amenable to treatment and therefore, immunotherapy can be a rational approach for patients with variable disease burdens. Last, there are other biomarkers which may more accurately reflect the true biologic response to treatment. These include circulating tumor cells (CTCs), as well as markers of bone turnover.

Despite the attempt of many vaccines to break immunologic tolerance, the overall failure to demonstrate clinical benefit despite induction of antibodies against the immunogen has remained a clinical challenge. Another issue has been the lack of immediacy of detecting clinical benefit; several recent trials in metastatic melanoma using a monoclonal antibody against checkpoint 
inhibitor CTLA-4 demonstrated an antitumor effect preceded initially by tumor growth then a remission. There still remains the unanswered question as to which clinical state would likely derive the best benefit from vaccines: the state of biochemical relapse where logically one is targeting micrometastatic disease or, noncastrate and castrate metastatic disease where one can actually follow the radiographic response to treatment. Where to position these new agents in the clinical state scheme continues to be a treatment challenge as more patients will likely be on these established agents earlier in the disease state while remaining stable for longer periods of time.

Sipuleucel-T is a cellular immunotherapy product that consists of an enriched population of a patient's peripheral blood mononuclear cells (PBMCs), which were exposed in vitro to a fusion protein comprised of granulocyte/macrophage-colony stimulating factor (GM-CSF) and prostatic acid phosphatase (PAP; Kantoff et al., 2010). These PMBCs, which include a large proportion of antigen-presenting cells, are infused back into the patient to stimulate antitumor T-cell responses (Kantoff et al., 2010). The specific indication of sipuleucel-T includes asymptomatic or minimally symptomatic, non-visceral, metastatic prostate cancer in the pre-docetaxel space.

Three phase III sipuleucel-T trials enrolled CRPC patients with only minor symptoms or functional impairment, and no current chemotherapy; however, $5.5,10.2$, and $18.2 \%$ of patients had prior chemotherapy (Higano et al., 2009; Kantoff et al., 2010). The trials reported an increase of 4.1-4.3 months in median overall survival compared with placebo. Estimated survival at 36 months was 32$33 \%$ in the active arms versus $15-23 \%$ in the placebo arms (Higano et al., 2009; Kantoff et al., 2010). However, early results of combination therapy with docetaxel have not demonstrated benefits of sipuleucel-T use following chemotherapy (Higano et al., 2009; Kantoff et al., 2010). These two analyses focused on the treatment benefit derived from the administration of sipuleucel-T with or without subsequent docetaxel. In the first two phase III trials, 32\% of the participants received docetaxel after either sipuleucel-T or a similar product prepared from their frozen cells (which was given to crossover placebo recipients after disease progression; Petrylak et al., 2007; Higano et al., 2009; Petrylak, 2011).

An exploratory post hoc analysis of docetaxel with or without early sipuleucel-T found that there was a benefit to receiving docetaxel some months after sipuleucel-T. Median survival was 34.5 months for patients who received sipuleucel-T followed later by docetaxel $(N=51)$; 25.7 months for crossover placebo recipients who also received docetaxel $(N=21)$; and 20.2 months for placebo patients who received docetaxel without ever receiving a vaccine product $(N=10)$. The adjusted survival hazard ratio (HR) for the first of these groups compared with the others was 2.53 ( $P=0.006$; Petrylak et al., 2007; Petrylak, 2011).

\section{RATIONALE FOR COMBINING CHEMO- AND IMMUNOTHERAPIES DO COMBOS ENHANCE EFFICACY VIA IMMUNOLOGIC MODULATION OR VIA TRUE BIOLOGIC (ANTITUMOR) EFFECT?}

Although an attractive idea, chemotherapy-vaccine combinations have not been widely applied. Much of the chemotherapy/vaccine combination data are from preclinical and murine studies, or are based on small phase I trials. In mouse models of colon and breast cancer, paclitaxel, docetaxel, or cisplatin subsequent to vaccination enhanced the effectiveness of the vaccine-generated cytotoxic lymphocytes (CTLs), probably by causing an increase in tumor cell permeability to granzyme B (Ramakrishnan et al., 2010). Cell death in the vaccinated and treated mouse cancer models included a desirable bystander effect in which the vaccine-induced CTLs caused apoptosis in neighboring tumor cells not expressing the vaccine antigens.

While the docetaxel used after early sipuleucel- $T$ was an exploratory study with small numbers, the results, while inconclusive, suggest that the potential to impact on disease with combination therapy is feasible. A number of authors have noted that natural cell-mediated immune responses (i.e., tumor infiltration by IFN $\gamma$-producing CD4+ and CD $8+$ cells) correlate with improved survival in a variety of cancers (Pagès et al., 2008; Mlecnik et al., 2011; Vesely et al., 2011) and some reports have even suggested that the type and density of infiltrating lymphocytes are more predictive of disease progression than traditional tumor staging (Pagès et al., 2008; Mlecnik et al., 2011).

However, it is felt that the chronic inflammatory milieu that develops during tumor growth compromises the immune response while promoting further progression of the malignancy (Wu and Zhou, 2009; Grivennikov et al., 2010; Vesely et al., 2011). Chronic inflammation causes immune cells to release cytokines such as TNF- $\alpha$, TGF- $\beta$, and IL-6, which recruit myeloid-derived suppressor cells (MDSC) and directly reduce immune cell activity. These cytokines also promote metastatic transition later in the disease process.

In addition, tumor cells evade CTLs through a number of strategies, including blocking antigen presentation, loss of MHC, and apoptosis (Poschke et al., 2011; Vesely et al., 2011). They can also produce immunosuppressive cytokines such as TGF- $\beta$ and VEGF. These cytokines directly reduce CTL numbers and recruit CD25+ Treg cells and MDSC that repress the immune response. Yet another mechanism employed by tumor cells may cause reduction in CTL numbers by expressing certain receptor ligands (e.g., PD-L1 and FasL).

There is considerable evidence in prostate cancer that tumors promote immune tolerance starting early in the disease (Diener et al., 2009; Drake, 2010). In a transgenic mouse model of prostate cancer, CD8+ and CD4+ cells specific to prostate antigens infiltrate prostate tumors but are anergic or non-functional. The encounter with tumor antigens apparently shifts CD4+ and CD8+ cells toward a suppressive (Treg) phenotype. Patient biopsies show that prostate tumor infiltrating CD4+ cells include high levels of Treg cells (Sfanos et al., 2008). Human prostate tumors also contain elevated populations of possibly protective Th17 cells populations, but only in early disease (low-grade tumors; Sfanos et al., 2008).

\section{IS THERE A LINK BETWEEN ANTI-CANCER IMMUNITY AND RESPONSE TO CHEMOTHERAPY?}

Chemotherapy is widely held to be immunosuppressive, but in fact, it has immunomodulatory effects (Zitvogel et al., 2008; Ma et al., 2011). Merely debulking the tumors reverses tumor-induced immune tolerance, possibly through reducing the amount of suppressive cytokines secreted by malignant cells (Zitvogel et al., 
2008). In addition, the transient lymphopenia caused by properly dosed chemotherapy activates homeostatic mechanisms, eliminating excess suppressor cells, and stimulating tumor-specific effector T-cell proliferation as well as dendritic cell maturation (McDonnell et al., 2011).

Some chemotherapeutic agents promote specific immune cell types. For example, docetaxel administration in a mouse model selectively decreased MDSCs while increasing CTL responses (Kodumudi et al., 2010). Docetaxel may have a relatively potent effect, but other taxanes also alter cytokine patterns and enhance lymphocyte proliferation, as well as the cytotoxic activity of NK and LAK cells, while reducing Treg cell populations (Javeed et al., 2009; Vicari et al., 2009).

Another murine study immunized mice with implanted colon tumors expressing human carcinoembryonic antigen (CEA). The experimental vaccine was based on a poxvirus vaccine containing genes for CEA and costimulatory molecules (CEA-TRICOM; Garnett et al., 2008). Administering a standard dose of docetaxel 4 days after 2 poxvirus immunizations improved vaccinespecific immune responses. It also induced antigen-specific T-cell responses to tumor-derived antigens distinct from the antigen used in the vaccine (the "antigen cascade" or "epitope spreading" possibly due to the release of antigens from dying cells). Docetaxel was effective only when administered after immunization. If administered beforehand, docetaxel inhibited cellular infection by the viral vaccine or antigen expression in the cells that did become infected (Garnett et al., 2008).

The optimal dosing of chemotherapy combined with vaccines remains unclear. A study that investigated daily low-dose paclitaxel found that by targeting HPV E7+ implanted tumors in mice receiving a DNA vaccine, survival was extended and tumor growth, delayed. The results were improved when compared with the vaccine alone, the vaccine plus high-dose paclitaxel, or high-dose, twice-weekly paclitaxel alone (Chen et al., 2010). Daily low-dose paclitaxel did not result in the significant $\mathrm{T}$-cell declines induced by high-dose paclitaxel. When administered with the vaccine, daily low-dose paclitaxel resulted in a higher CD8+ T-cell/Treg ratio than either the vaccine alone or the vaccine plus highdose paclitaxel. Furthermore, the low-dose chemotherapy had greater anti-angiogenic effects than did high-dose (Chen et al., 2010).

Low-dose cyclophosphamide also has well-documented modulatory effects (Sistigu et al., 2011). It reduces the Treg population, inhibits the activity of the remaining Treg cells, and stimulates cell-mediated immunity (Garnett et al., 2008). The drug is now reappearing in vaccine trials after a hiatus of many years. In a transgenic murine prostate cancer model, administering lowdose cyclophosphamide 1-2 days before immunization with a whole-cell, GM-CSF-secreting vaccine (GVAX) resulted in a tumor shrinkage effect not observed with the vaccine alone (Wada et al., 2009). This effect seemed related to a reduced Treg population in the tumor and its draining lymph node as well as increased dendritic cell activation (Wada et al., 2009).

Other studies have found specific benefits from high-dose but submyeloablative chemotherapy. An adenovirus-based vaccine was found to have limited effectiveness in mice with established melanoma tumors unless the mice were pretreated with higher doses of cyclophosphamide (Grinshtein et al., 2009). The combination resulted in tumor regression due to the high frequency of vaccine antigen-specific T cells, reflecting cyclophosphamide's general promotion of cell-mediated immunity. Another approach used cisplatin/vinorelbine to induce leukopenia as well as downmodulated reconstitution of Treg cells when compared with effector T cells. Gameiro et al. (2011) and Noguchi et al. (2004, 2011), have presented their experience of using low-dose estramustine phosphate (initially administered as $280 \mathrm{mg}$ daily in concert with a personalized peptide vaccine in HLA-A2 ${ }^{+}$or $-\mathrm{A} 24^{+}$patients with CRPC (Noguchi et al., 2004). Other than good tolerability and feasibility of administration, there were no preclinical studies to suggest that this drug had a direct immunomodulatory effect. Nevertheless, 10 of 11 patients who received a combination of peptide vaccination and estramustine showed a serum PSA decrease; 8 patients showed a PSA decrease of $\geq 50 \%$. One of two patients with measureable disease showed a $44 \%$ decrease in lymph node metastasis; no changes were seen in patients with bone lesions. Estramustine-induced immunosuppression was analyzed in 10 of 11 patients by IFN- $\gamma$ productions to $\mathrm{PHA}$, EBV peptide and the vaccinated peptides. Immunologic responses were observed; no significant immune suppression was seen when the peptide was given along with a smaller dose of estramustine (Noguchi et al., 2004). These responses were thought to be mediated by resting $\mathrm{T}$ cell, memory $\mathrm{T}$ cells, and a combination of memory and activated T cells, respectively (Noguchi et al., 2004). These observations were further extended into another phase I multicenter study (Noguchi et al., 2011) of 15 patients treated with los dose estramustine and ITK-1, a peptide set consisting of 14 kinds of peptide which induced HLA-A24-resetricted tumor-specific cytotoxic activity. Patient were treated with the top four peptides shown to be immunogenic based on pre-vaccination measurement of peptide-specific IgG in plasma reactive to ITK-1. While safe, in vitro analysis did not reveal any correlation between the peptide dose and the generation of specific $\mathrm{T}$ cells from vaccinated patients. A median survival of 23.8 months was reported with this combination regimen albeit the exact immune mechanism (Noguchi et al., 2011), if any, which contributed to this remains unclear.

\section{CAN REDUCING OR SUPPRESSING THE REGULATORY T-CELL POPULATION WITH NON-CHEMOTHERAPEUTIC AGENTS ENHANCE THE RESPONSE TO VACCINE?}

Improving vaccine response by inactivating Treg cells has been attempted through specific targeting of the T-cell checkpoint inhibitor, CTLA-4 with a monoclonal antibody such as ipilimumab (Noguchi et al., 2004, 2011; Saha and Chatterjee, 2010). Preliminary clinical trials suggest that administering a therapeutic vaccine followed by ipilimumab enhances immune responses and tumor reduction in prostate and ovarian cancers as well as melanoma (Chakraborty et al., 2007; Li et al., 2007; Gerritsen et al., 2008; Mohebtash et al., 2009). In a non-comparative phase I trial $(N=30)$ of ipilimumab plus the PSA-TRICOM vaccine in prostate cancer, overall survival was 31.8 months compared with an expected survival of 18.5 months based on baseline factors (Halabi nomogram-predicted survival, HPS; Hodi et al., 2008; Yuan et al., 2008). 
Several studies in melanoma have not found additional benefit for therapeutic vaccines beyond that of ipilimumab alone (Halabi et al., 2003; Madan et al., 2010a). In both the prostate cancer and melanoma studies, the vaccines were administered simultaneously with the course of ipilimumab; there was no attempt to evaluate sequential therapy (Halabi et al., 2003; Madan et al., 2010a). Furthermore, the melanoma studies associated ipilimumab with autoimmune events such as diarrhea, hypophysitis, transaminitis, and rash, as did a prostate cancer study of ipilimumab alone after radiotherapy (Mohebtash et al., 2009; Sarnaik et al., 2011). The severity of the adverse events appeared to be related to the level of response to ipilimumab. Overall, the results suggest that chemotherapy, albeit at less than therapeutic doses, may induce a wide range of immune effects which may ultimately lead to a systemic antitumor effect, albeit several weeks to months after treatment. Another checkpoint inhibitor, programmed death 1 (PD-1), PD-1 is a member of the extended CD28/CTLA-4 family of T-cell regulators. Monoclonal antibodies to PD-1 are currently in clinical trials and have shown promise in renal cell carcinoma, lung cancer, and melanoma. Combinatorial trials with anti-CTLA-4 and other immune modulatory agents are currently ongoing.

In a hypothesis-driven pilot study based on a preclinical tumorbearing model of mice immunized with peptide pulse dendritic cells followed by an anti-VEGF antibody, patients with biochemically relapsed prostate cancer were treated with three doses of Sipuleucel-T followed by every 2 week infusions with Bevacizumab at $10 \mathrm{mg} / \mathrm{kg}$ (Rini et al., 2006). The preclinical data suggested a more robust and durable antitumor immune response in those mice treated with dendritic cells and Bevacizumab compared with peptide-pulsed DC alone. Several patients had modulation of PSA and immunologic monitoring suggested brisk T-cell proliferation which was associated with a $>50 \%$ PSA decline in one patient. All patients had increased production of interferon- $\gamma$ by EliSPOT assay (Rini et al., 2006). Though a very small patient cohort was studied, this work opened a new perspective, introducing the possibility of using biologic agents such as VEGF inhibitors or even cytokines in combinatorial approaches with immunotherapy.

\section{CAN COMBINATION TRIALS DEMONSTRATE AN IMPACT ON TUMOR RESPONSE OR OVERALL SURVIVAL IN PROSTATE CANCER?}

Recent attempts to demonstrate improvement in overall or progression-free survival (PFS) have been shown using a poxvirusPSA recombinant vaccine, a mixture of recombinant pox viruses expressing either PSA or the B7.1 costimulatory molecule (Gameiro et al., 2011). A vaccinia-based vaccine is usually administered once followed by monthly injections of fowlpox-PSA recombinant virus in a prime boost strategy. Each vaccination is administered with GM-CSF (Arlen et al., 2006). In a phase II study, patients with metastatic CRPC $(N=28)$ received the poxvirus-PSA vaccine 2 weeks apart for the first month and then monthly until disease progression occurred. Half the group additionally received docetaxel/dexamethasone therapy in 3-weeks-on/1-week-off cycles (Arlen et al., 2006). Eleven patients (78.6\%) in the vaccine-alone arm were changed to docetaxel upon evidence of progression. Median time to progression was 1.8 months in the vaccine-alone arm and 3.2 months in the vaccine plus docetaxel arm. Notably, patients experienced a median 6.1-month progression-free period after progressing on the vaccine alone and then switching to docetaxel. PFS was 3.7 months in an historic control group receiving docetaxel alone (Arlen et al., 2006). All evaluable vaccine recipients exhibited increased PSA-specific T cells (median 3.33-fold increase in both arms). In the three vaccine-alone recipients who were examined, T-cell responses emerged, as well as responses to other prostate tumor antigens (PAP, PSMA, and/or MUC-1). This may have been due to an epitope-spreading phenomenon pursuant to the tumor cell death by the vaccine-induced PSA-specific T-cell response (Arlen et al., 2006).

ProstVac VF (PSA-TRICOM) is a second-generation vaccine employing recombinant vaccinia- and fowlpox-expressing PSA plus 3 T-cell costimulatory molecules, LFA-3, B7.1, and ICAM-1 (Poschke et al., 2011). It elicits a more robust antitumor response than the original poxvirus-PSA immunization (Poschke et al., 2011). An ongoing randomized double-blind placebo-controlled, multicenter phase III efficacy trial of PROSTVAC in men with asymptomatic or minimally symptomatic, CMPC is a 3-arm study and will evaluate overall survival in two separate comparisons, PROSTVAC plus adjuvant dose GM-CSF versus controls, and PROSTVAC without GM-CSF versus controls: (1) (Arm $\mathrm{V}+\mathrm{G}$ ) PROSTVAC-V/F plus adjuvant dose GM-CSF; (2) (Arm V) PROSTVAC-V/F plus GM-CSF placebo; (3) (Arm P) Double placebo (vector placebo plus GM-CSF placebo).

Prostate GVAX is a polyvalent vaccine comprised of irradiated whole cells from two prostate cancer cell lines, the androgendependent LNCaP and the androgen-refractory PC3, respectively (Drake, 2010). In this vaccine, the cells are transduced with a gene for GM-CSF in order to improve their immune-stimulatory effects (Drake, 2010). Phase II studies showed promising responses and led to two phase III studies that included docetaxel. The phase III VITAL-1 trial directly compared GVAX (biweekly for the first 26 weeks, then monthly) with standard tri-weekly docetaxel plus prednisone (Higano et al., 2009). The study population included chemotherapy-naïve men with CPRC and negligible pain. This trial was terminated early due to futility: there was little chance of reaching the primary endpoint, improved survival, even though indications of the vaccine efficacy were observed. Median survival was 20.7 months on GVAX and 21.7 months on docetaxel/prednisone $(P=0.78)$. Of note, grade $3 / 4$ adverse events were considerably less frequent with GVAX ( $8.8 \%$ of GVAX recipients vs $43 \%$ of those on docetaxel; Higano et al., 2009).

The futility analysis took on particular importance because of the results in the other phase III GVAX trial (VITAL-2). That study administered docetaxel every 3 weeks followed 2 days later by GVAX immunization. After 10 docetaxel cycles, GVAX every 4 weeks was administered alone as maintenance therapy. The comparator group received standard docetaxel/prednisone for 10 cycles (Small et al., 2009). The study had a planned enrollment of 600 taxane-naïve patients with metastatic CRPC requiring opioid pain management; overall survival was the primary endpoint (Small et al., 2009). However, the trial $(N=408$ actual enrollment) was halted prematurely due to an excess of deaths in the GVAX arm (67 vs 47). Median overall survival was 12.2 months in the GVAX/docetaxel arm and 14.1 months in the docetaxel/prednisone arms $(\mathrm{HR}=1.70 ; 95 \% \mathrm{CI}, 1.15-2.53$, 
$P=0.0076)$. The investigators were unable to identify safety issues or other reasons for the excess deaths.

It should be noted that no phase II trials were conducted prior to VITAL-2 in order to test various docetaxel/GVAX doses and sequences. Judging by the VITAL-1 results with GVAX alone, it is conceivable that the concurrent high-dose docetaxel undercut the GVAX effect. Administering GVAX before or after docetaxel rather than concurrently might yield a more successful result. Another possibility is that some of the study population had disease that was too advanced to benefit from the vaccine.

\section{CONCLUSION: HOW SHOULD INVESTIGATORS GO FORWARD WITH IMMUNOTHERAPY TRIALS?}

There remain at least three questions which remain unaddressed with respect to chemotherapy administered with an immunotherapy: (1) what will be the most effective chemotherapy, (2) what is the most appropriate to foster synergism, and (3) what is the sequence of reagent delivery to foster maximum benefit? Chemotherapies with potential for benefit include the taxanes, anthracyclines, and cyclophosphamide but it remains unclear whether newer targeting agents against the androgen receptor (AR), or signaling pathways may also play a role. All of these agents appear to have positive immunomodulation in and of themselves that might enhance the response to a therapeutic vaccine. As discussed earlier, chemotherapy given in lower-than-therapeutic doses may be favored, as those may selectively alter cell populations and inhibit angiogenesis (Chen et al., 2010; Emmenegger et al., 2011; Sistigu et al., 2011). Higher doses may permit greater immune activity and more immunogenicity due to tumor debulking and cell death (Ma et al., 2011). Lower doses and/or abbreviated courses would be less toxic overall, and also less immunosuppressive. They would also allow frequent, even daily, dosing (metronomic administration) for a steady effect over time.

Sequencing of chemotherapy, as in dosing, depends on the agent's mechanism of action. Initiation of chemotherapy prior to vaccination would be an option if the goal was to reset the immune system by reducing the level of suppressive cells. Conversely, initiation of chemotherapy during or after vaccination would be an option if the strategy was to impede the tumor and potentiate or broaden the vaccine-induced responses.

The question of which patient, disease stage, and treatment history is most appropriate for therapeutic vaccine schemes also

\section{REFERENCES}

Arlen, P. M., Gulley, J. L., Parker, C., Skarupa, L., Pazdur, M., Panicali, D., Beetham, P., Tsang, K. Y., Grosenbach, D. W., Feldman, J., Steinberg, S. M., Jones, E., Chen, C., Marte, J., Schlom, J., and Dahut, W. (2006). A randomized phase II study of concurrent docetaxel plus vaccine versus vaccine alone in metastatic androgen-independent prostate cancer. Clin. Cancer Res. 12, 1260-1269.

Beer, T. M., Slovin, S. F., Higano, C. S., Tejwani, S., Dorff, T. B., Stankevich, E., Lowy, I., and Prostate Cancer Clinical Trials Consortium.
(2008). Phase I trial of ipilimumab (IPI) alone and in combination with radiotherapy (XRT) in patients with metastatic castration resistant prostate cancer (mCRPC). J. Clin. Oncol. 26, abstr. 5004.

Chakraborty, M., Schlom, J., and Hodge, J. W. (2007). The combined activation of positive costimulatory signals with modulation of a negative costimulatory signal for the enhancement of vaccine-mediated T-cell responses. Cancer Immunol. Immunother. 56, 1471-1484.

Chen, C. A., Ho, C. M., Chang, M. C., Sun, W. Z., Chen, Y. L., Chiang, Y. C., Syu, M. H., Hsieh, C. Y., and

arises. Late-stage patients may have had their immune systems compromised by extensive chemotherapy and the evolving tumor escape strategies (Higano et al., 2009). One implication is that the patients with shorter life expectancies will not benefit from vaccine therapy; for example, as demonstrated in the GVAX/docetaxel combination results (Beer et al., 2008). Considering the safety of vaccines relative to standard chemotherapy, clinical trials could justify enrolling patients in earlier stages of disease in lieu of conventional chemotherapy alone.

\section{CAN VACCINE TRIAL ENDPOINTS BE IMPROVED?}

Another reason for suboptimal results with vaccines in populations with advanced disease and low life expectancies is that the timeframe needed to observe a clinical response may be delayed. Researchers have realized that responses to immunotherapies are slower compared to chemotherapy (Wolchok et al., 2009; Hoos et al., 2010; Madan et al., 2010b; Emmenegger et al., 2011). The disease could remain stable or even progress for some months before protective immune responses are apparent. Alternatively, the initial vaccine-induced inflammatory flare may be mistaken for tumor growth. Various groups have therefore proposed revised endpoints for cancer vaccine trials that place greater emphasis on overall survival or long-term disease stability rather than PFS. The emphasis is on minimizing premature discontinuation, and allowing patients to continue with therapy despite early, minor progression (Hoos et al., 2010; Emmenegger et al., 2011).

These endpoints may be more appropriate, but they unfortunately codify the extension of vaccine/chemotherapy combination studies' length and complexity. This further compromises an area that historically has been of low research priority. Biomarkers of immune response that reliably predict treatment outcome would simplify researchers issues, allowing for more rapid identification and the optimization of effective regimens before trials have reached clinical endpoints (Hoos et al., 2007; Disis, 2011; Emmenegger et al., 2011). These would ideally give advance indication of clinical benefit without the long followup required to observe clinical endpoints. In general, biomarkers, there remains the question as to whether blood biomarkers reflect conditions within the tumor itself of within the surrounding milieu. There is also a need to standardize immune assays so that study results become more easily reproducible (Hoos et al., 2010).

Cheng, W. F. (2010). Metronomic chemotherapy enhances antitumor effects of cancer vaccine by depleting regulatory $\mathrm{T}$ lymphocytes and inhibiting tumor angiogenesis. Mol. Ther. 18, 1233-1243.

Diener, K. R., Woods, A. E., Manavis, J., Brown, M. P., and Hayball, J. D. (2009). Transforming growth factor-beta-mediated signaling in $\mathrm{T}$ lymphocytes impacts on prostatespecific immunity and early prostate tumor progression. Lab. Invest. 89 , 142-151.

Disis, M. L. (2011). Immunologic biomarkers as correlates of clinical response to cancer immunotherapy.
Cancer Immunol. Immunother. 60, 433-442.

Drake, C. G. (2010). Prostate cancer as a model for tumour immunotherapy. Nat. Rev. Immunol. 10, 580-593.

Emmenegger, U., Francia, G., Chow, A., Shaked, Y., Kouri, A., Man, S., and Kerbel, R. S. (2011). Tumors that acquire resistance to low-dose metronomic cyclophosphamide retain sensitivity to maximum tolerated dose cyclophosphamide. Neoplasia 13, 40-48.

Gameiro, S. R., Caballero, J. A., Higgins, J. P., Apelian, D., and Hodge, J. W. (2011). Exploitation of differential homeostatic proliferation of $\mathrm{T}$-cell 
subsets following chemotherapy to enhance the efficacy of vaccinemediated antitumor responses Cancer Immunol. Immunother. 60 , 1227-1242.

Garnett, C. T., Schlom, J., and Hodge, J. W. (2008). Combination of docetaxel and recombinant vaccine enhances T-cell responses and antitumor activity: effects of docetaxel on immune enhancement. Clin. Cancer Res. 14, 3536-3544.

Gerritsen, W., van den Eertwegh, A. J., de Gruijl, T., Giaccone, G., Scheper, R. J., Lowy, I., Levy, E., Hege, K., and Sacks, N. (2008). Expanded phase I combination trial of GVAX immunotherapy for prostate cancer and ipilimumab in patients with metastatic hormone-refractory prostate cancer (mHPRC). J. Clin. Oncol. 26, abstr. 5146.

Grinshtein, N., Ventresca, M., Margl, R., Bernard, D., Yang, T. C., Millar, J. B., Hummel, J., Beermann, F., Wan, Y., and Bramson, J. L. (2009). Highdose chemotherapy augments the efficacy of recombinant adenovirus vaccines and improves the therapeutic outcome. Cancer Gene Ther. 16, 338-350.

Grivennikov, S. I., Greten, F. R., and Karin, M. (2010). Immunity, inflammation, and cancer. Cell 140, 883-899.

Halabi, S., Small, E. J., Kantoff, P. W., Kattan, M. W., Kaplan, E. B., Dawson, N. A., Levine, E. G., Blumenstein, B. A., and Vogelzang, N. J. (2003). Prognostic model for predicting survival in men with hormone-refractory metastatic prostate cancer J. Clin. Oncol. 21, 1232-1237.

Higano, D., Saad, F., Somer, B., Curti, B., Petrylak, D., Drake, C. G., Schnell, F., Redfern, C. H., Schrijvers, D., and Sacks, N. (2009). "A phase III trial of GVAX immunotherapy for porstate cancer versus docetaxel plus prednisone in asymptomatic, castrationresistant prostate cancer (CRPC)," in 2009 Genitourinary Cancers Symposium, Abstract LBA150, Orlando, FL.

Hodi, F. S., Butler, M., Oble, D. A., Seiden, M. V., Haluska, F. G., Kruse, A., MacRae, S., Nelson, M., Canning, C., Lowy, I., Korman, A., Lautz, D., Russell, S., Jaklitsch, M. T., Ramaiya, N., Chen, T. C., Neuberg, D., Allison, J. P., Mihm, M. C., and Dranoff, G. (2008). Immunologic and clinical effects of antibody blockade of cytotoxic T lymphocyte-associated antigen 4 in previously vaccinated cancer patients. Proc. Natl. Acad. Sci. U.S.A. 105, 3005-3010.
Hodi, F. S., O’Day, S. J., McDermott, D. F., Weber, R. W., Sosman, J. A., Haanen, J. B., Gonzalez, R., Robert, C., Schadendorf, D., Hassel, J. C., Akerley, W., van den Eertwegh, A. J., Lutzky, J., Lorigan, P., Vaubel, J. M., Linette, G. P., Hogg, D., Ottensmeier, C. H., Lebbé, C., Peschel, C., Quirt, I., Clark, J. I., Wolchok, J. D., Weber, J. S., Tian, J., Yellin, M. J., Nichol, G. M., Hoos, A., and Urba, W. J. (2010). Improved survival with ipilimumab in patients with metastatic melanoma. N. Engl. J. Med. 363, 711-723.

Hoos, A., Eggermont, A. M., Janetzki, S., Hodi, F. S., Ibrahim, R., Anderson, A., Humphrey, R., Blumenstein, B., Old, L., and Wolchok, J. (2010). Improved endpoints for cancer immunotherapy trials. J. Natl. Cancer Inst. 102, 1388-1397.

Hoos, A., Parmiani, G., Hege, K., Sznol, M., Loibner, H., Eggermont, A., Urba, W., Blumenstein, B., Sacks, N., Keilholz, U., Nichol, G., and Cancer Vaccine Clinical Trial Working Group. (2007). A clinical development paradigm for cancer vaccines and related biologics. J. Immunother. 30, 1-15.

Javeed, A., Ashraf, M., Riaz, A., Ghafoor, A., Afzal, S., and Mukhtar, M. M. (2009). Paclitaxel and immune system. Eur. J. Pharm. Sci. 38, 283-290.

Kantoff, P. W., Higano, C. S., Shore, N. D., Berger, E. R., Small, E. J., Penson, D. F., Redfern, C. H., Ferrari, A. C., Dreicer, R., Sims, R. B., Xu, Y., Frohlich, M. W., Schellhammer, P. F., and IMPACT Study Investigators. (2010). Sipuleucel-T immunotherapy for castration-resistant prostate cancer. N. Engl. J. Med. 363, 411-422.

Kodumudi, K. N., Woan, K., Gilvary, D. L., Sahakian, E., Wei, S., and Djeu, J. Y. (2010). A novel chemoimmunomodulating property of docetaxel: suppression of myeloidderived suppressor cells in tumor bearers. Clin. Cancer Res. 16, 4583-4594.

Li, N., Qin, H., Li, X., Zhou, C., Wang, D., Ma, W., Lin, C., Zhang, Y., Wang, S., and Zhang, S. (2007). Synergistic antitumor effect of chemotacticprostate tumor-associated antigen gene-modified tumor cell vaccine and anti-CTLA-4 mAb in murine tumor model. Immunol. Lett. 113, 90-98.

Ma, Y., Conforti, R., Aymeric, L., Locher, C., Kepp, O., Kroemer, G. C. J., DeMarzo, A. M., Meeker, A. K., Isaacs, W. B., Drake, C. G., and Zitvogel, L. (2011). How to improve the immunogenicity of chemotherapy and radiotherapy. Cancer Metastasis Rev. 30, 71-82.

Madan, R. A., Mohebtash, M., Arlen, P. M., Rauckhorst, M., Ferrara, T. A., Steinberg, S. M., Dahut, W. L., Schlom, J., and Gulley, J. L. (2010a). Overall survival (OS) analysis of a phase l trial of a vector-based vaccine (PSA-TRICOM) and ipilimumab (Ipi) in the treatment of metastatic castration-resistant prostate cancer (mCRPC). J. Clin. Oncol. 28, abstr. 2550.

Madan, R. A., Gulley, J. L., Fojo, T., and Dahut, W. L. (2010b). Therapeutic cancer vaccines in prostate cancer: the paradox of improved survival without changes in time to progression. Oncologist 15, 969-975.

McDonnell, A. M., Nowak, A. K. and Lake, R. A. (2011). Contribution of the immune system to the chemotherapeutic response. Semin. Immunopathol. 33, 353-367.

Mlecnik, B., Bindea, G., Pages, F., and Galon, J. (2011). Tumor immunosurveillance in human cancers. Cancer Metastasis Rev. 30, 5-12.

Mohebtash, M., Madan, R. A., Arlen, P. M., Rauckhorst, M., Tsang, K. Y., Cereda, V., Vergati, M., Poole, D. J., Dahut, W. L., Schlom, J., Gulley, J. L., and National Cancer Institute, Bethesda, MD (2009). Phase I trial of targeted therapy with PSA-TRICOM vaccine (V) and ipilimumab (ipi) in patients (pts) with metastatic castration-resistant prostate cancer (mCRPC). J. Clin. Oncol. 27, abstr. 5144.

Noguchi, M., Itoh, K., Suekane, S., Morinaga, A., Sukehiro, A., Suetsugu, N., Katagiri, K., Yamada, A., and Noda, S. (2004). Immunological monitoring during combination of patientoriented peptide vaccination and estramustine phosphate in patients with metastatic hormone refractory prostate cancer. Prostate 60, 32-45.

Noguchi, M., Uemura, H., Naito, S., Akaza, H., Yamada, A., and Itoh, K. (2011). A phase I study of personalized peptide vaccination using 14 kinds of vaccine in combination with low-dose estramustine in HLA-A24-positive patients with castration-resistant prostate cancer. Prostate 71, 470-479.

Pagès, F., Galon, J., and Fridman, W. H. (2008). The essential role of the in situ immune reaction in human colorectal cancer. J. Leukoc. Biol. 84, 981-987.

Petrylak, D. (2011). "Defining the optimal role of immunotherapy and chemotherapy," in Chemotherapy Foundation Symposium XXIV2006. Available at: http://www.mssmtv.org/player/ player.php?id=111006ada_petrylak. [accessed August 24].

Petrylak, D. P., Schellhammer, P. F., Small, E., and Frohlich, M. W. (2007). "Androgen independent prostate cancer (AIPC) patients who receive sipuleucel- $T$ followed by docetaxel have prolonged survival," in American Urology Association Meeting Abstracts, Orlando, FL, 605.

Poschke, I., Mougiakakos, D., and Kiessling, R. (2011). Camouflage and sabotage: tumor escape from the immune system. Cancer Immunol. Immunother. 60, 1161-1171.

Ramakrishnan, R., Assudani, D., Nagaraj, S., Hunter, T., Cho, H. I., Antonia, S., Altiok, S., Celis, E., and Gabrilovich, D. I. (2010). Chemotherapy enhances tumor cell susceptibility to CTL-mediated killing during cancer immunotherapy in mice. J. Clin. Invest. 120, 1111-1124.

Rini, B. I., Weinber, V., Fong, L., Conry, S., Hershberg, R. M., and Small, E. J. (2006). Combination immunotherapy with prostatic acid phosphatase pulsed antigen-presenting cells (Provenge) plus bevacizumab in patients with serologic progression of prostate cancer after definitive local therapy. Cancer 107, 68-74.

Saha, A., and Chatterjee, S. K. (2010). Combination of CTL-associated antigen-4 blockade and depletion of CD25 regulatory $\mathrm{T}$ cells enhance tumour immunity of dendritic cellbased vaccine in a mouse model of colon cancer. Scand. J. Immunol. 71, 70-82.

Sarnaik, A. A., Yu, B., Yu, D., Morelli, D., Hall, M., Bogle, D., Yan, L., Targan, S., Solomon, J., Nichol, G., Yellin, M., and Weber, J. S. (2011). Extended dose ipilimumab with a peptide vaccine: immune correlates associated with clinical benefit in patients with resected high-risk stage IIIc/IV melanoma. Clin. Cancer Res. 17, 896-906.

Sfanos, K. S., Bruno, T. C., Maris, C. H., Xu, L., Thoburn, C. J., DeMarzo, A. M., Meeker, A. K., Isaacs, W. B., and Drake, C. G. (2008). Phenotypic analysis of prostate-infiltrating lymphocytes reveals TH17 and Treg skewing. Clin. Cancer Res. 14, 3254-3261.

Sistigu, A., Viaud, S., Chaput, N., Bracci, L., Proietti, E., and Zitvogel, L. (2011). Immunomodulatory effects of cyclophosphamide and implementations for vaccine design. Semin. Immunopathol. 33, 369-383. 
Slovin, S. F. (2008). Tribulations or triumphs in prostate cancer immunotherpy: on the road to victory? Expert Rev. Anticancer Ther. 8 465-474.

Small, E., Demkow, T., Gerritsen, W. R., Rolland, F., Hoskin, P., Smith, D. C., Parker, C., Chondros, D., Ma, J., and Hege, K. (2009). "A phase III trial of GVAX immunotherapy for prostate cancer in combination with docetaxel versus docetaxel plus prednisone in symptomatic, castrationresistant prostate cancer (CRPC)," in 2009 Genitourinary Cancers Symposium, Orlando, Abstract LBA150.

Vesely, M. D., Kershaw, M. H., Schreiber, R. D., and Smyth, M. J. (2011). Natural innate and adaptive immunity to cancer. Annu. Rev. Immunol. 29, 235-271.

Vicari, A. P., Luu, R., Zhang, N., Patel, S., Makinen, S. R., Hanson, D. C., Weeratna, R. D., and Krieg, A. M. (2009). Paclitaxel reduces regulatory $\mathrm{T}$ cell numbers and inhibitory function and enhances the anti-tumor effects of the TLR9 agonist PF-3512676 in the mouse. Cancer Immunol. Immunother. 58, 615-628.

Wada, S., Yoshimura, K., Hipkiss, E. L., Harris, T. J., Yen, H. R., Goldberg, M. V., Grosso, J. F., Getnet, D., Demarzo, A. M., Netto, G. J., Anders, R., Pardoll, D. M., and Drake, C. G. (2009). Cyclophosphamide augments antitumor immunity: studies in an autochthonous prostate cancer model. Cancer Res. 69, 4309-4318.

Wolchok, J. D., Hoos, A., O’Day, S., Weber, J. S., Hamid, O., Lebb, C., Maio, M., Binder, M., Bohnsack, O., Nichol, G., Humphrey, R., and Hodi, F. S. (2009). Guidelines for the evaluation of immune therapy activity in solid tumors: immune-related response criteria. Clin. Cancer Res. 15, 7412-7420.

Wu, Y., and Zhou, B. P. (2009). Inflammation: a driving force speeds cancer metastasis. Cell Cycle 8, 3267-3273.

Yuan, J., Gnjatic, S., Li, H., Powel, S., Gallardo, H. F., Ritter, E., Ku, G. Y., Jungbluth, A. A., Segal, N. H., Rasalan, T. S., Manukian, G., $\mathrm{Xu}$, Y., Roman, R. A., Terzulli, S. L., Heywood, M., Pogoriler, E., Ritter, G., Old, L. J., Allison, J. P., and Wolchok, J. D. (2008). CTLA-4 blockade enhances polyfunctional NY-ESO-1 specific T cell responses in metastatic melanoma patients with clinical benefit. Proc. Natl. Acad. Sci. U.S.A. 105, 20410-20415.

Zitvogel, L., Apetoh, L., Ghiringhelli, F., Andre, F., Tesniere, A., and Kroemer, G. (2008). The anticancer immune response: indispensable for therapeutic success? J. Clin. Invest. 118 , 1991-2001.

Conflict of Interest Statement: The author declares that the research was conducted in the absence of any commercial or financial relationships that could be construed as a potential conflict of interest.

Received: 10 January 2012; paperpending published: 03 February 2012; accepted: 12 April 2012; published online: 30 May 2012.

Citation: Slovin SR (2012) Toward maximizing immunotherapy in metastatic castration-resistant prostate cancer rationale for combinatorial approaches using chemotherapy. Front. Oncol. 2:43. doi: 10.3389/fonc.2012.00043

This article was submitted to Frontier in Genitourinary Oncology, a specialty of Frontiers in Oncology.

Copyright (c) 2012 Slovin. This is an open-access article distributed under the terms of the Creative Commons Attribution Non Commercial License, which permits non-commercial use, distribution, and reproduction in other forums, provided the original authors and source are credited. 